\title{
Nobel Lecture: Interplay of disorder and interaction in two-dimensional electron gas in intense magnetic fields*
}

\author{
Daniel C. Tsui \\ Department of Electrical Engineering, Princeton University, Princeton, New Jersey 08544
}

[S0034-6861(99)00804-1]

In this lecture, I would like to briefly go through the physics that I have learned in the years since I ventured into what we nowadays call research of semiconductor electronics in low dimensions, or in my case, more simply the electronic properties of two-dimensional systems (Ando et al., 1983). To summarize, electrons confined to the interface of two different semiconductors normally behave like an ordinary gas of particles in two dimensions. But, when taken to extreme conditions of low temperature and high magnetic field, they show new physics phenomena manifesting the interplay of electron-electron interactions and the interaction of the electrons with imperfections in the semiconductors. Let me first recall the events in my earlier research that led me to the journey that Art Gossard, Horst Stormer, and I took in our adventure towards the discovery of the fractional quantum Hall effect (FQHE) (Tsui et al., 1982).

\section{PROLOGUE}

I joined Bell Laboratories in the spring of 1968. I was sufficiently naive that I foolhardily convinced myself to leave behind the more familiar band structures and Fermi surfaces of metal physics, which I had become comfortable with through my years of research as a graduate student, and to try something different, e.g., surfaces or interfaces, and preferably some many-body interaction physics. I read about Anderson localization, the Mott transition, and the notion that disorder and electron-electron interaction were the richest and most challenging problems in solid-state physics. But I did not have the foggiest idea on how to do what to get started.

Fortunately, I was advised to talk to John Rowell, who had at the time just completed the RowellMcMillan electron-phonon interaction work using tunneling in superconductivity. John told me to look into point-contact tunneling into the high- $T_{c}$ superconductors of those days and suggested that, instead of using tungsten whiskers, I should experiment with semiconductor tips which have built-in surface depletion layers as tunnel barriers. My effort to tunnel into the superconductors of niobium and vanadium compounds was unsuccessful, but the project forced me to learn some physics of semiconductor surfaces and interfaces. In fact, I

\footnotetext{
*The 1998 Nobel Prize in Physics was shared by Robert B. Laughlin, Horst L. Stormer, and Daniel C. Tsui. This lecture is the text of Professor Tsui's address on the occasion of the award.
}

was able to demonstrate experimentally the electric-field quantization of the surface space-charge layer, first proposed by Schrieffer in the fifties, by doing a tunneling experiment on InAs to observe directly the quantized energy levels and the Landau levels of the resulting twodimensional (2D) electrons (Tsui, 1970). However, the most exciting part of this effort was my discovery, in writing the paper on this work, of the beautiful work on the Si metal-oxide-semiconductor field-effect transistor ( $\mathrm{Si}$ MOSFET) done by the IBM group in Yorktown Heights (Fang et al., 1983). They laid a solid foundation for the development and growth of $2 \mathrm{D}$ electron physics in the subsequent decades.

Based on the IBM work, Jim Allen and I made a temperature dependence study of the inversion layer conductance in Si MOSFETs to look for the 2D Anderson localization-delocalization transition. By varying the gate voltage on the device, we were able to move the Fermi level into the band tail and observe directly a transition from the two-dimensional electron gas (2DEG) behavior to the behavior of an insulator. But, to our disappointment, we were unable to obtain quantitative agreement with theory. We thought interaction might be the cause and came to the conclusion that to enhance the interaction we should apply a magnetic field, which would change the individual electron's kinetic energy into the cyclotron energy. This should be especially effective in the extreme quantum limit when the cyclotron diameter was less than the average electron-electron separation.

In a 1976 paper, Kawaji and Wakabayashi (1976) reported the observation of localized states in the energy gap between two Landau levels. This discovery was a most important milestone on the path to the quantized Hall effect. In response to their work, I studied at the Frances Bitter National Magnet Laboratory on the MIT campus the conductance in the extreme quantum limit, when all electrons occupy the lowest Landau level, and saw some structures. Phil Anderson, after hearing this from John Rowell, asked to see the data. But by the time I showed them to him in the Bell Labs tearoom, I had repeated the experiment and found them to be sample specific. I told this to Phil and he made a cryptic remark under his breath that there should be some commensuration energy anyway. I reasoned: given that $n$ is the $2 \mathrm{D}$ electron density and that the magnetic field $B$ (applied perpendicular to the 2D plane) is expressed in terms of average flux density $n_{\phi}=B / \varphi_{0}$ (and $\varphi_{0}=h / e$ is the Dirac flux quantum), the ratio $n / n_{\varphi}$ is the Landaulevel filling factor $\nu$. For $n>n_{\varphi}$, an integer $i$ number of Landau levels are filled at commensuration and the cy- 


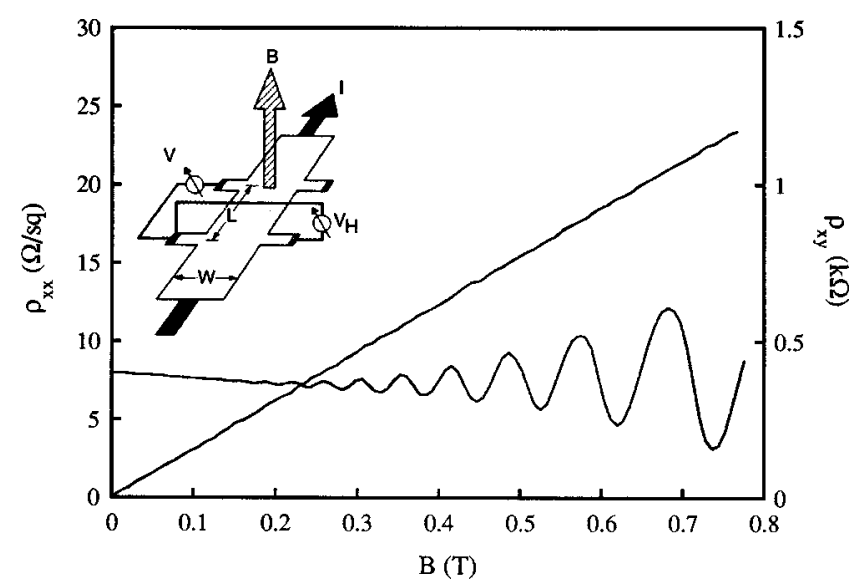

FIG. 1. Magneto-transport coefficients $\rho_{x x}$ and $\rho_{x y}$ of a 2DEG in $\mathrm{GaAs} / \mathrm{Al}_{x} \mathrm{Ga}_{1-x} \mathrm{As}$ at $0.35 \mathrm{~K}$ in moderately low $B$. The insert shows the measurement geometry. The magnetic field $B$ is perpendicular to the plane of the 2DEG and to the current $I$. The voltages $V$ and $V_{H}$ are measured along and perpendicular to $I$, respectively, $\rho_{x x}=(V / L) /(I / W)$ is the resistance across a square, independent of the square size, and $\rho_{x y}=V_{H} / I$ is the Hall resistance independent of the sample width. Data taken by A. Majumdar.

clotron energy, separating the filled from the empty levels, is the commensuration energy. I assumed he meant: In the $n<n_{\varphi}$ extreme quantum limit, at commensuration when $\nu=n / n_{\varphi}=1 / i$, some interaction energy might become dominant to drive the $2 \mathrm{D}$ system into some new ground state. I was not brave enough to ask him: "What do you mean?" But I felt affirmed that I should continue to concentrate on the extreme quantum limit.

Indeed, with the advent of molecular beam epitaxy (Cho, 1995) and the invention of modulation doping to produce highly perfect 2D electron systems (Stormer et al., 1979), it soon became quite clear to Art Gossard, Horst Stormer, and me that where we wanted to go to look for new many-body interaction physics should be a highest-mobility 2DEG sample placed in a most intense magnetic field.

\section{TWO-DIMENSIONAL MAGNETO-TRANSPORT}

In the presence of a perpendicular magnetic field, the energy levels of a two-dimensional electron collapse, as a result of Landau quantization of its cyclotron orbits, into discrete Landau levels separated by the cyclotron energy quantum. Scattering broadens the Landau levels and gives rise to $2 \mathrm{D}$ magneto-transport described by the Ando-Uemura theory (Ando and Uemura, 1979). Figure 1 is an example showing the quantum oscillations in the diagonal resistivity $\rho_{x x}$, reflecting the broadened Landau-level structure of the 2DEG, and the Hall resistance $\rho_{x y}$, well known from the Drude model. However, when the $2 \mathrm{DEG}$ is taken to the extreme condition of high $B$ and low $T$, much more striking features appear, showing the interplay of disorder and electron-electron interaction in the system. More specifically, different physics phenomena are observed in three distinctly dif-

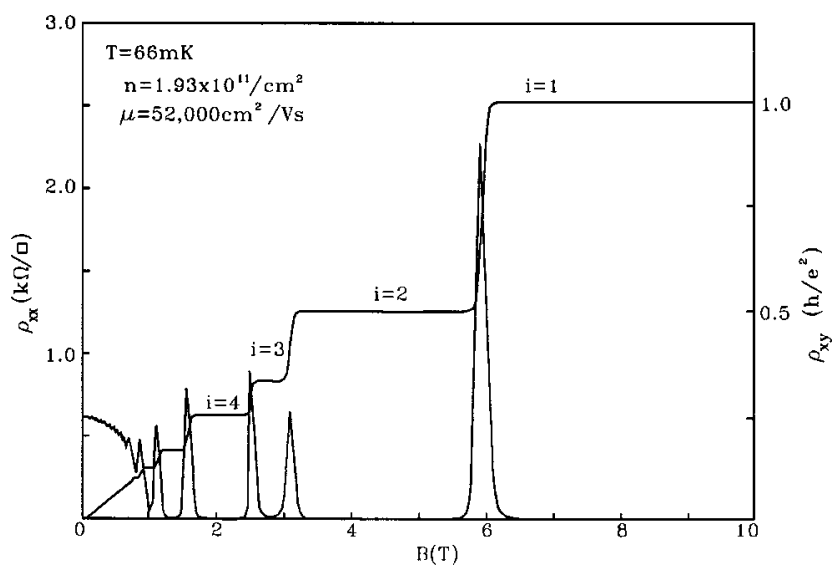

FIG. 2. $\rho_{x x}$ and $\rho_{x y}$ of a relatively low-mobility $2 \mathrm{DEG}$ in $\mathrm{GaAs} / \mathrm{Al}_{x} \mathrm{Ga}_{1-x}$ As. The plateaus in $\rho_{x y}$ are quantized in the natural conductance unit $e^{2} / h$ with integer quantum numbers $i=1,2, \ldots$. Data taken by H. P. Wei.

ferent physical regimes. The first is the disorderdominant regime, when the sample is dirty with low 2D electron mobility (e.g., $\mu<10^{5} \mathrm{~cm}^{2} / \mathrm{V} \mathrm{sec}$ in the case of GaAs). The striking features in the data constitute the integral quantum Hall effect (IQHE) (von Klitzing et al., 1980), which is understood in terms of the physics of independent electrons and their localization in the presence of random impurities in the semiconductors. The fractional quantum Hall effect (FQHE) is observed in high-mobility samples in the regime where the electronelectron interaction dominates. It manifests the manybody interaction physics of the $2 \mathrm{DEG}$ in the intense $B$ field. Furthermore, even in the cleanest samples, the FQHE series terminates into an insulator in the high- $B$ limit. This insulator is believed to be an electron crystal pinned by defects to the semiconductor. The third regime is this high- $\mu$ and high- $B$-field limit, where disorder and interaction play equally important roles and need to be treated on an equal footing.

\section{QUANTUM PHASE TRANSITIONS IN IQHE}

Quantization of the Hall resistance in the natural conductance unit $e^{2} / h$ is currently understood in terms of the existence of an energy gap, separating the excited states from the ground state, and localized states inside the gap. In the IQHE case, where the quantum numbers are integers identified with the number of completely filled Landau levels, the energy gap is the Landau gap of a cyclotron energy quantum. The accurate quantization was shown by Laughlin, using a gedanken experiment, to be a consequence of charge quantization. He showed that the experiment in effect measures the charge carried by the excited electron. The localized states arise from disorder in the 2D system, and the data, as shown in Fig. 2, show the localization-delocalization phase transitions. In other words, for $B$ in the plateau regions, the states at $E_{F}$ are localized, and in between, delocalized. As $T$ is decreased, the range of $B$ for the existence of delocalized states decreases and the transition regions 


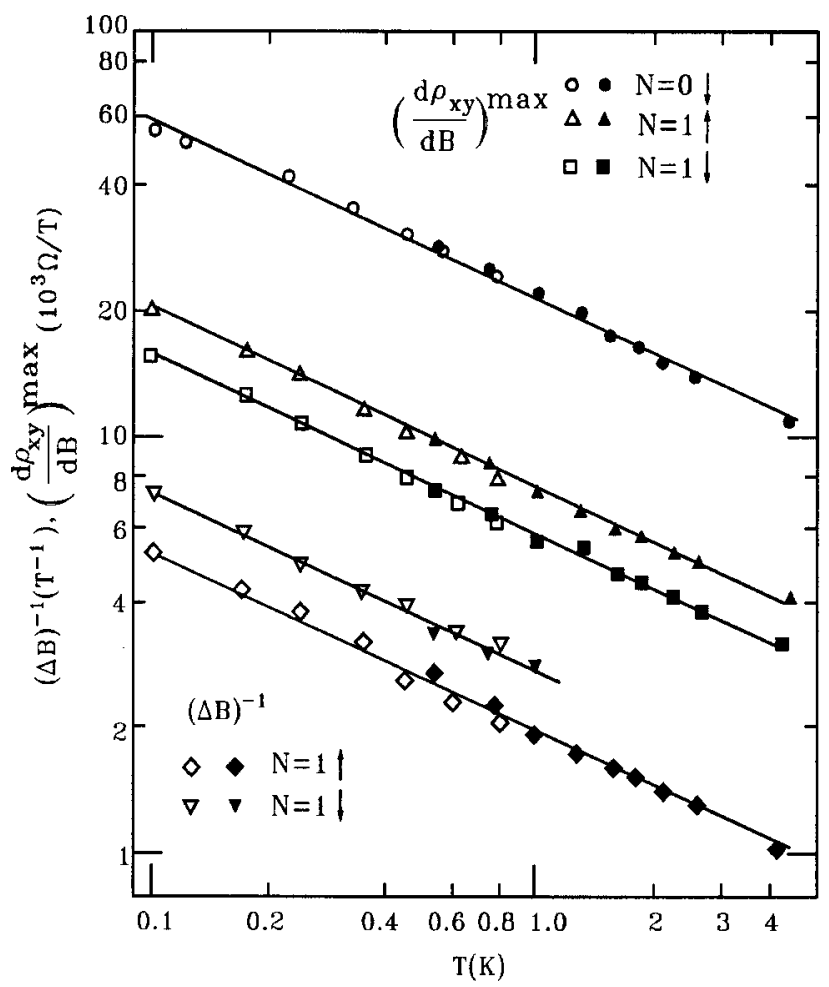

FIG. 3. Data on narrowing of the plateau-to-plateau transition regions from an $\operatorname{In}_{x} \mathrm{Ga}_{1-x} \mathrm{As} / \mathrm{InP}$ sample with $n=3.3$ $\times 10^{11} / \mathrm{cm}^{2}$ and $\mu=3.4 \times 10^{4} \mathrm{~cm}^{2} / \mathrm{V} \mathrm{s}$. The upper portion shows the maximum in $\left(d \rho_{x y} / d B\right)$ for the $i=1 \rightarrow 2,2 \rightarrow 3$, and $3 \rightarrow 4$ transitions (i.e., the $N=0 \downarrow, 1 \uparrow$, and $1 \downarrow$, respectively); the lower portion shows the inverse half-width $(\Delta B)^{-1}$ of the $\rho_{x x}$ peak for the $i=2 \rightarrow 3$ and $3 \rightarrow 4$ transitions. $N$ is the Landaulevel quantum number. From Wei et al., 1988.

between the plateaus narrow. In the limit $T \rightarrow 0, \rho_{x y}$ approaches a staircase. The underlying physics is the Anderson localization-delocalization quantum phase transition, and the experiment is simply a magnificent display of 2D quantum critical phenomena, as first put forward by Pruisken (1985).

Quantum phase transitions take place at $T=0$. To relate them to experiments relies on the finite $T$ behavior of the system at sufficiently close to the transition, which is governed by special rules, derived from simple scaling arguments. In our case, this boils down to narrowing of the plateau-to-plateau transition regions following a power-law dependence on $T$, with the temperature exponent a universal constant. Figure 3 shows data from Wei et al. (1988), where the narrowing of the transition regions is measured by the maximum in $d \rho_{x y} / d B$ and by the inverse half width $(\Delta B)^{-1}$ of the $\rho_{x x}$ peak. The data show a power-law dependence $\sim T^{-\kappa}$ and the temperature exponent $\kappa=0.42 \pm 0.04$, independent of the Hall plateaus involved. Furthermore, the hallmark of a quantum phase transition is that the finite frequency $f$ behavior of the system and its finite $T$ behavior are similar: both follow a power-law dependence and are characterized by the same exponent. Crossover from one to the other occurs around $h f=k T$. Engel et al. (1993) studied the microwave conductance in the frequency range from $0.2 \mathrm{GHz}$ to $16 \mathrm{GHz}$ in a dilution refrigerator and were

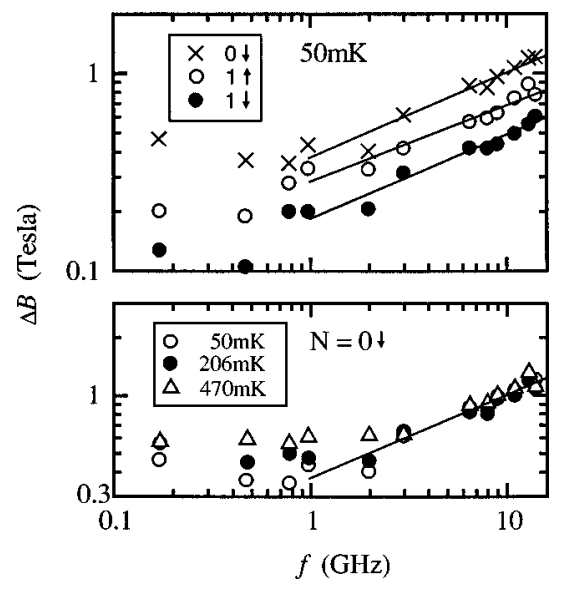

FIG. 4. Microwave frequency dependence of the width of the plateau-to-plateau transition regions. Upper panel: Half-width $\Delta B$ of $\sigma_{x x}(f)$ for the $i=1 \rightarrow 2,2 \rightarrow 3$, and $3 \rightarrow 4$ transitions (i.e., the $N=0 \downarrow, N=1 \uparrow$, and $N=1 \downarrow$, respectively). Lines are leastsquares fits of data for $f \geqslant 0.97 \mathrm{GHz}$ to $\Delta B \sim f^{\gamma}$, with $\gamma=0.43$, 0.38 , and 0.42 , respectively. Lower panel: $\Delta B$ of $\sigma_{x x}(f)$ for the $i=1 \rightarrow 2$ transition at three different $T$ 's. The line is a fit to $\Delta B \sim f^{0.43}$. From Engel et al., 1993.

able to go from $f<k T / h$, where the scaling is dominated by $T$, to $f>k T / h$, where frequency scaling should hold. Their data are shown in Fig. 4. They show a power-law dependence on $f$ in the $f>k T / h$ limit with a frequency exponent equal to the $T$ exponent within the experimental error bar, and are consistent with a crossover around $h f=k T$.

\section{THE FQHE}

The second regime, where the electron-electron interaction dominates, is accessible using high-mobility samples. In this regime, the FQHE becomes observable, and a large number of plateaus in $\rho_{x y}$ and concomitant $\rho_{x x}$ minima are apparent in the data (Figs. 5 and 6), even after the IQHE structures are exhausted in the $\nu<1$ extreme quantum limit. These plateaus, as determined from their resistance values, are fractionally quantized, and they occur around the same fractions of Landaulevel filling. At such fractional fillings, the singleelectron levels are highly degenerate and there is no energy gap across $E_{F}$ to possibly give rise to Hall resistance quantization. Horst Stormer and Bob Laughlin will discuss the new many-body interaction physics manifested in the phenomenon and the broader implications of it in their lectures. Here, I simply want to mention the so-called odd-denominator rule, that all the fraction quantum numbers are odd-denominator fractions, and to point out that there is now a firmly established exception at $\nu=5 / 2$. Over a decade ago, Willett et al. (1987) reported the observation of a deep minimum in $\rho_{x x}$ and a clear deviation of the Hall resistance from its classical line around $\nu=5 / 2$ filling, suggestive of an even-denominator fraction 5/2 FQHE. Very recently, Wei Pan and Jian-Sheng Xia, working with the University of Florida micro-kelvin group in Gainesville, cooled 


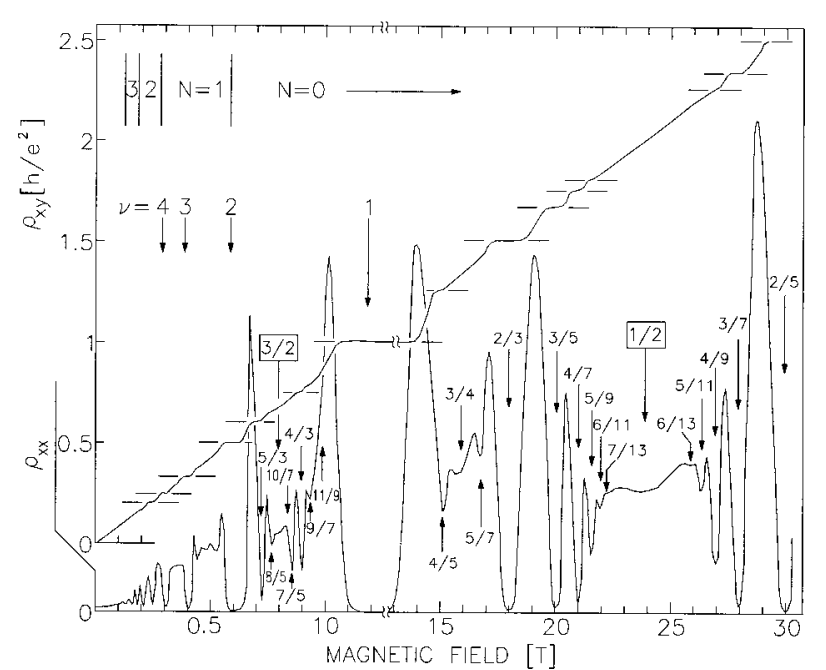

FIG. 5. $\rho_{x x}$ and $\rho_{x y}$ of a $2 \mathrm{DEG}$ in $\mathrm{GaAs} / \mathrm{Al}_{x} \mathrm{Ga}_{1-x} \mathrm{As}$ with $n$ $=3.0 \times 10^{11} / \mathrm{cm}^{2}$ and $\mu=1.3 \times 10^{6} \mathrm{~cm}^{2} / \mathrm{V} \mathrm{s}$. The fractionally quantized Hall resistance plateaus are indicated by the horizontal bars and the odd-denominator fractions marking the concomitant vanishing $\rho_{x x}$. The use of a hybrid magnet with fixed base field required composition of this figure from four different traces (breaks at $\simeq 12 \mathrm{~T}$ ). Temperatures were $\approx 150$ $\mathrm{mK}$ except for the high-field Hall trace at $T=85 \mathrm{mK}$. The high-field $\rho_{x x}$ trace is reduced in amplitude by a factor of 2.5 for clarity. $N$ is the Landau-level quantum number. Filling factor $\nu$ is indicated. From Willett et al., 1987.

the 2DEG below $10 \mathrm{mK}$ and were able to observe a Hall plateau quantized to better than two parts in $10^{6}$, thus making the FQHE nature of the ground state at $\nu=5 / 2$ unequivocal.

\section{THE MAGNETIC-FIELD-INDUCED CRYSTAL REGIME}

Finally, there is the third regime, where disorder and interaction are equally important. This is the small-
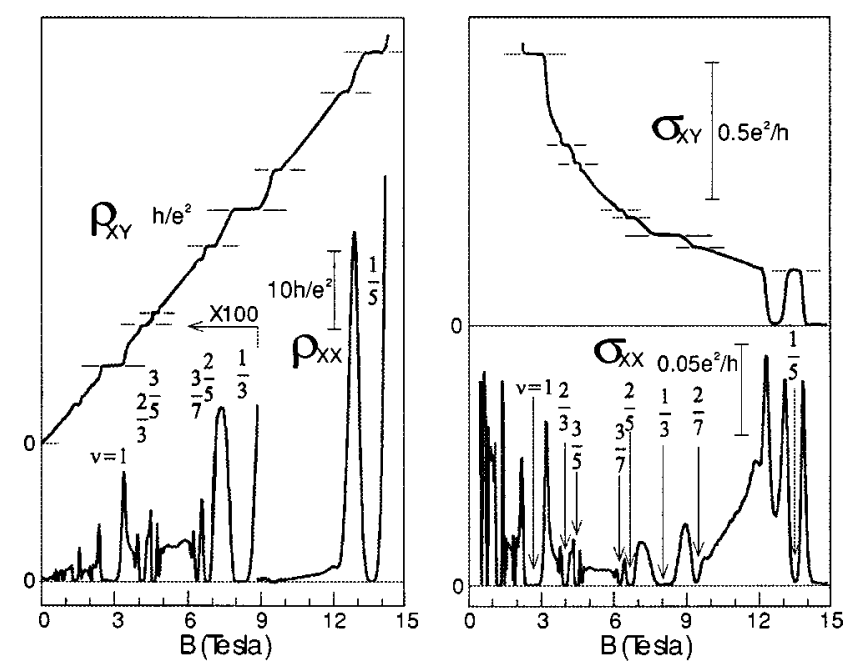

FIG. 6. Magneto-transport coefficients of a 2DEG in $\mathrm{GaAs} / \mathrm{Al}_{x} \mathrm{Ga}_{1-x} \mathrm{As}$ with $n=6.5 \times 10^{10} / \mathrm{cm}^{2}$ and $\mu=1.5$ $\times 10^{6} \mathrm{~cm}^{2} / \mathrm{V}$ s. Left panel: $\rho_{x x}$ and $\rho_{x y}$ at $40 \mathrm{mK}$. Right panel: $\sigma_{x x}$ and $\sigma_{x y}$ obtained by inverting the $\rho_{x x}$ and $\rho_{x y}$ data. The vanishing of $\sigma_{x y}$ together with $\sigma_{x x}$ at $B \sim 12.8 \mathrm{~T}$ and $B>14 \mathrm{~T}$ indicates insulating behavior. Data taken by Y. P. Li.
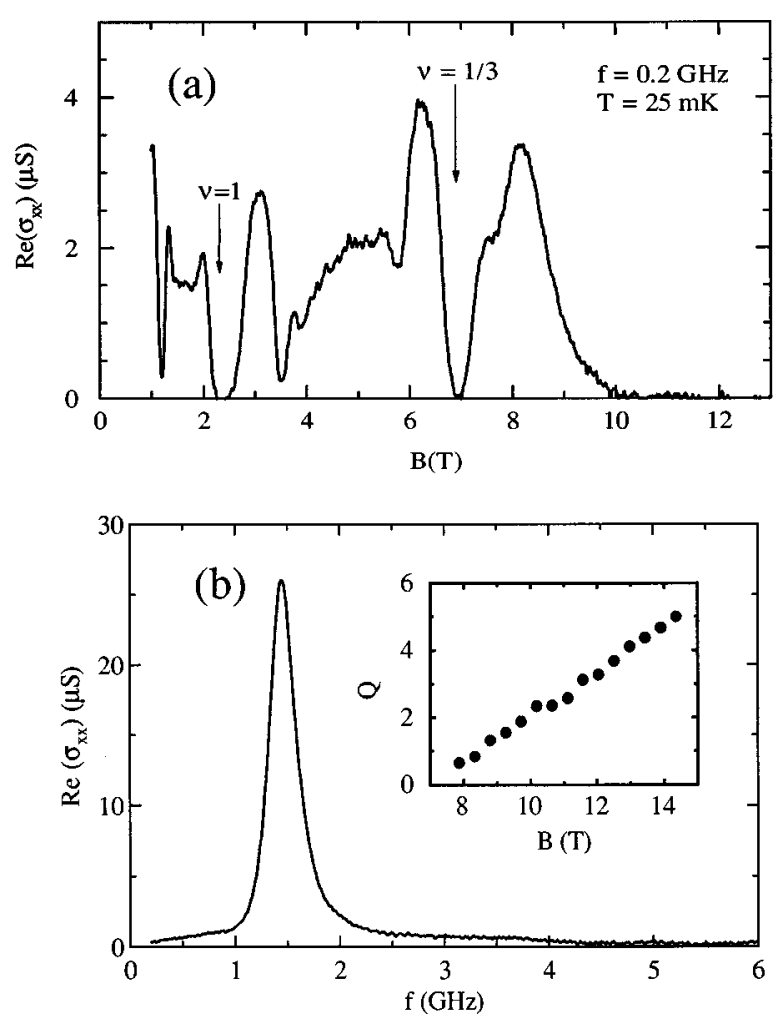

FIG. 7. Behavior of a two-dimensional hole gas (2DHG): (a) Real part of diagonal conductivity vs magnetic field of a twodimensional hole gas in $\mathrm{GaAs} / \mathrm{Al}_{x} \mathrm{Ga}_{1-x}$ As with a hole density of $5.5 \times 10^{10} / \mathrm{cm}^{2}$ and $\mu=3.5 \times 10^{5} \mathrm{~cm}^{2} / \mathrm{V} \mathrm{s}$. The $2 \mathrm{DHG}$ becomes insulating for $B \geq 10 \mathrm{~T}$. (b) Resonant microwave absorption in the insulating phase of the $2 \mathrm{DHG}$ at $B=13 \mathrm{~T}$ and $T=25 \mathrm{mK}$. The insert shows the quality factor of the resonance $Q$ vs $B$. From Li et al., 1997.

filling limit after the FQHE series terminates into an insulating phase. In the absence of disorder, the ideal $2 \mathrm{DEG}$ is predicted to be a $2 \mathrm{D}$ electron crystal at sufficiently small fillings. But, in real physical systems, there is always disorder that can alter the ground state in fundamental ways. To date, insulating behavior is seen in the highest-mobility 2D electrons for $\nu<1 / 5$, and 2D holes for $\nu<1 / 3$. This insulating phase in the cleanest 2D systems we have is attributed to crystallization of the $2 \mathrm{D}$ electron and 2D hole gases under an intense B field. The crystal, being pinned to the semiconductor by defects in the semiconductor, cannot slide to conduct electricity.

Experimentally, this is a challenging regime. It requires all the best: the highest-mobility samples, lowest $T$, and most intense $B$. Since it is an insulator, dc transport is limited. Microwave measurements, more appropriate at first sight, are notoriously hard and can be plagued with pitfalls. Consequently, unequivocal experiments are few and there is very little direct information on the crystalline nature of the ground state. A great many of the properties of this insulating phase still remain unknown and unexplored. Chi-Chun Li and Lloyd Engel (Li et al., 1997) recently improved their microwave absorption experiment and obtained data (Fig. 7) showing a sharp conductance resonance in the insulating phase at $\sim 1.5 \mathrm{GHz}$, a frequency consistent with that expected of a pinning mode of the crystal. It is surprising, 
however, that the resonance is sharp, with a quality factor $Q$ much larger than 1 . The $Q$, as seen in the inset of Fig. 7(b), increases with increasing $B$ to the highest $B$ studied. Thus it appears that there is nontrivial fundamental physics hidden in this regime, and I look forward to further experimental efforts.

\section{ACKNOWLEDGMENTS}

In conclusion, I must emphasize that the FQHE journey that Horst Stormer and I have been on would never have gotten started without Art Gossard's participation in the early stage. To continue the journey to this day would also not have been possible without the hard work, the intellectual stimulation, and the youthful vitality of our graduate students and postdoctoral associates, and the contributions and collaborations of our colleagues in Bell Laboratories, Princeton University, and elsewhere in our community. They include K. W. Baldwin, P. Berglund, G. S. Boebinger, A. M. Chang, R. Du, J. P. Eisenstein, L. W. Engel, V. J. Goldman, M. Grayson, M. Hilke, H. W. Jiang, G. Kaminsky, C. Li, Y. P. Li, M. A. Paalanen, W. Pan, A. Pruisken, T. Sajoto, D. Shahar, J. A. Simmons, Y. W. Suen, H. P. Wei, R. Willett, and A. Yeh. Also, the most valuable support that made it possible for me to continue a research enterprise after moving to Princeton University was from the materials expert friends, K. Alavi, A. Y. Cho, J. E. Cunningham, J. H. English, A. C. Gossard, J. C. M. Hwang, J. F. Klem, R. A. Logan, L. N. Pfeiffer, M. Razeghi, M. B.
Santos, M. Shayegan, W. T. Tsang, C. Tu, G. Weimann, K. W. West, and W. Wiegmann, who taught me the basics of semiconductor materials and technology and often provided samples for us to experiment with.

\section{REFERENCES}

Ando, T., A. B. Fowler, and F. Stern, 1983, Rev. Mod. Phys. 54, 437.

Ando, T., and Y. Uemura, 1974, J. Phys. Soc. Jpn. 36, 959.

Cho, A. Y., 1995, MRS Bull. 20, 21.

Engel, L. W., D. Shahar, C. Kurdak, and D. C. Tsui, 1993, Phys. Rev. Lett. 71, 2638.

Fang, F. F., A. B. Fowler, W. E. Howard, F. Stern, P. J. Stiles, and collaborators, reviewed in Ando et al., Rev. Mod. Phys. 54, 437 (1983).

Kawaji, S., and J. Wakabayashi, 1976, Surf. Sci. 58, 238.

Li, C.-C., L. W. Engel, D. Shahar, D. C. Tsui, and M. Shayegan, 1997, Phys. Rev. Lett. 79, 1353.

Pruisken, A. M. M., 1985, Phys. Rev. B 32, 2636.

Stormer, H. L., R. Dingle, A. C. Gossard, W. Wiegmann, and M. Sturge, 1979, Solid State Commun. 29, 705.

Tsui, D. C., 1970, Phys. Rev. Lett. 24, 303.

Tsui, D. C., H. L. Stormer, and A. C. Gossard, 1982, Phys. Rev. Lett. 48, 1559.

von Klitzing, K., G. Dorda, and M. Pepper, 1980, Phys. Rev. Lett. 45, 494.

Wei, H. P., D. C. Tsui, M. A. Paalanen, and A. M. M. Pruisken, 1988, Phys. Rev. Lett. 61, 1294.

Willett, R., J. P. Eisenstein, H. L. Stormer, D. C. Tsui, A. C. Gossard, and J. H. English, 1987, Phys. Rev. Lett. 59, 1776. 